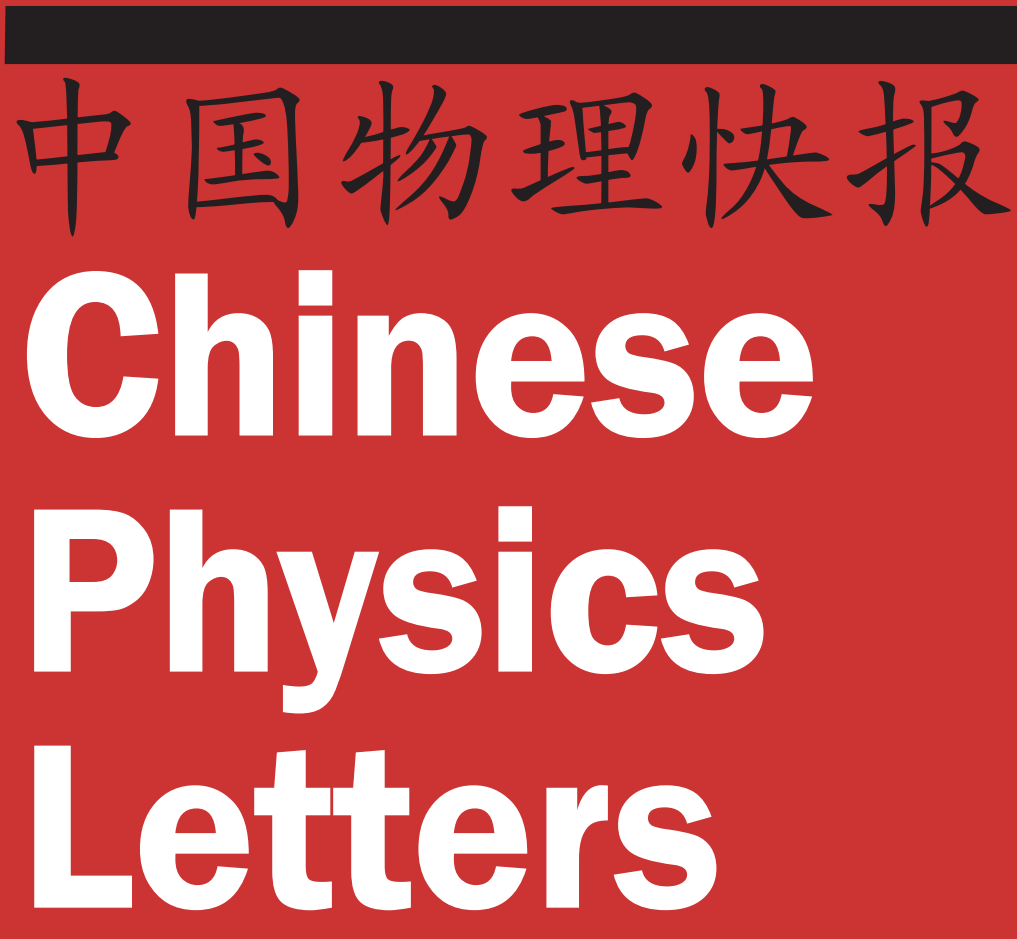

Volume 30 Number 5 May 2013

A Series Journal of the Chinese Physical Society Distributed by IOP Publishing

Online: http://iopscience.iop.org/0256-307X http://cpl.iphy.ac.cn

Chinese Physical Sóciety Institute of Physics greishing 


\title{
Visualization of a Maze-Like Reconstruction of Graphene on a Copper Surface at the Atomic Scale*
}

\author{
XIE Nan(谢楠 $)^{1,2}$, GONG Hui-Qi(宮会期 $)^{2}$, ZHOU Zhi(周智 $)^{2}$, GUO Xiao-Dong(郭晓东) $)^{2}$, \\ YAN Shi-Chao(颜世超 $)^{2}$, SUN Qian(孙骞 $)^{1}$, XING Sirui(邢思锐 $)^{3}$, WU Wei (吴纬 $)^{3}$, \\ PEI Shin-shem(白先慎 $)^{3}$, BAO Jiming(包吉明 $)^{3}$, SHAN Xin-Yan(单欣岩 $)^{2}$, \\ GUO Yang (郭阳) $)^{2 * *}$, LU Xing-Hua(陆兴华) $)^{2 * *}$ \\ ${ }^{1}$ The MOE Key Laboratory of Weak-Light Nonlinear Photonics, Tianjin Key Laboratory of Photonics Materials and \\ Technology for Information Science, School of Physics, Nankai University, Tianjin 300071 \\ ${ }^{2}$ Beijing National Laboratory for Condensed Matter Physics, Institute of Physics, \\ Chinese Academy of Sciences, Beijing 100190 \\ ${ }^{3}$ Department of Electrical and Computer Engineering, University of Houston, Houston, TX 77204, USA
}

(Received 28 January 2013)

\begin{abstract}
Interaction with the substrate plays an essential role in determining the structure and electronic property of graphene supported by a surface. We observe a maze-like reconstruction pattern in graphene on flat copper foil. With functionalized scanning tunneling microscope tips, a triangular three-for-six structure of graphene and a mixed $(2 \sqrt{2} \times \sqrt{2}) R 45^{\circ}$ reconstruction of a $\mathrm{Cu}(100)$ surface are separately visualized at the atomic scale. Substrate-induced changes in the structure and electronic property are further illustrated by micro-Raman spectroscopy and scanning tunneling spectroscopy. This finding suggests a new method to effectively induce partial $s p^{3}$ hybridization in a single-layer graphene and therefore to tune its electronic property through interaction with the substrate.
\end{abstract}

PACS: 68.65.Pq, 68.47.De, 68.37.Ef

DOI: $10.1088 / 0256-307 \mathrm{X} / 30 / 5 / 056802$

With a single layer of carbon atoms arranged in a honeycomb lattice, ${ }^{[1-9]}$ graphene has become a prominent candidate for future applications, especially novel two-dimensional electronic devices, due to its unique linear energy dispersion at low energies. ${ }^{[10,11]}$ Significant research progress has been made to tune the electrical properties of graphene through electrical gates, ${ }^{[12]}$ dopants and defects, ${ }^{[13-16]}$ local strain, ${ }^{[17-19]}$ and substrates. ${ }^{[20-22]}$ Associated with the electronic property changes, a three-for-six configuration has been observed on wrinkles of exfoliated graphene layers ${ }^{[17,18,23]}$ and on strained graphene layers grown on highly corrugated copper surfaces. ${ }^{[19]}$ Some $s p^{3}$ hybridization is induced in such configurations to effectively open an energy gap and turn the graphene into a semiconductor or an insulator. ${ }^{[13-15]}$ The interaction between graphene and the underlying substrate is crucial in determining the structure transformation and modification of electrical properties in graphene. However, it remains a great challenge to fully understand the graphene-substrate interaction because of the difficulty in probing the atomic structure of the underlying substrate. In addition, the observation of new reconstruction patterns due to graphene-substrate interaction is strongly desired.

In this Letter, we present our investigation of a maze-like reconstruction in graphene on a copper surface by using a low-temperature scanning tunnel- ing microscope (STM). The reconstruction pattern is composed of a three-for-six triangular lattice and an orthogonal lattice, which are separately visualized with functionalized STM tips. The presence of partial $s p^{3}$ hybridization in the triangular lattice is verified by the appearance of a midgap state near the Fermi level in the local electronic density of states. The orthogonal lattice is illustrated as a mixed $(2 \sqrt{2} \times \sqrt{2}) R 45^{\circ}$ reconstruction of a $\mathrm{Cu}(100)$ surface as supported by $\mathrm{x}$-ray diffraction data. Our results suggest a strong interaction between the graphene and copper surface that has not been reported up until now.

The graphene sample used in this study is synthesized on $\mathrm{Cu}$ foil $(50-\mu \mathrm{m}$-thick) by the ambient chemical vapor deposition (CVD) method. ${ }^{[24,25]}$ The growth is carried out at around $1050^{\circ} \mathrm{C}$ with $\mathrm{CH}_{4}$ as the carbon stock. The sample is then further annealed at $400^{\circ} \mathrm{C}$ for $6 \mathrm{~h}$ in an ultra-high vacuum (UHV) chamber (base pressure $<10^{-10}$ Torr). A scanning electron microscope (SEM) and a home-built low-temperature STM are employed to characterize the sample surface. The local electronic density of states (LDOS) is measured by recording the STM differential conductance $(d I / d V)$ signal under open-loop feedback conditions. The sample bias is modulated at $361.1 \mathrm{~Hz}$ with an rms amplitude of $10 \mathrm{mV}$.

The SEM images in Fig. 1 show the morphology of the sample. The graphene flakes of a size around

\footnotetext{
*Supported by the National Natural Science Foundation of China under Grant Nos 11174347, 61027011 and 10974245, the National Basic Research Program of China under Grant No 2012CB933002, the Chinese Academic of Sciences under Grant No 1731300500030, the Robert a Welch Foundation (E-1728) and the National Science Foundation (DMR-0907336, ECCS-1240510). SSP, WW and SRX acknowledge support from the Delta Electronics Foundation and UH CAM.

** Corresponding author. Email: yangguo@iphy.ac.cn; xhlu@iphy.ac.cn

(C) 2013 Chinese Physical Society and IOP Publishing Ltd
} 
$10 \mu \mathrm{m}$ can be easily distinguished from the bare copper surface with characteristic hexagonal edges. Second layer graphene can been seen as smaller dark patches, which has been confirmed by micro-Raman studies. ${ }^{[25,26]}$ The surface morphology of the graphenecovered area is further illustrated by STM images at the nanometer scale. Two typical topographic features are observed. Some areas appear highly corrugated, as shown in Fig. 1(b), and some show ordered steps and flat terraces of micrometer size, as shown in Fig. 1(c), where the straight steps are several hundreds of nanometers in length and 3-5 atomic layers in height.
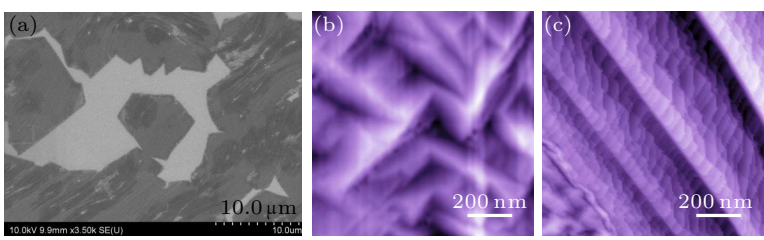

Fig. 1. The morphology of graphene on a copper surface. (a) SEM image of the sample, and (b) and (c) STM topographic images of graphene on a corrugated surface and on terraces and steps. The images $(1 \mu \mathrm{m} \times 1 \mu \mathrm{m})$ are taken with a sample bias of $-0.7 \mathrm{~V}$ and tunneling current of $0.1 \mathrm{nA}$ for (b), and $-0.5 \mathrm{~V}$ and $0.1 \mathrm{nA}$ for (c).
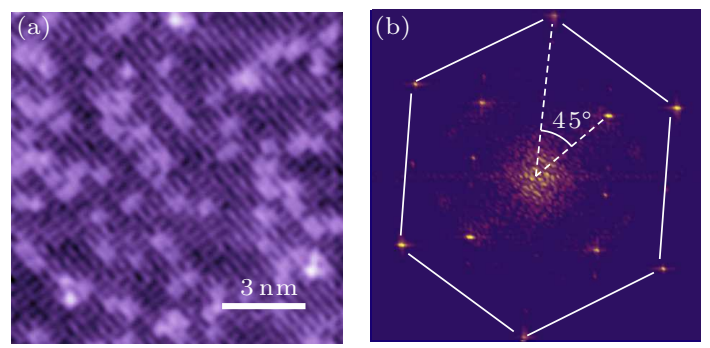

Fig. 2. (a) STM topographic image of a maze-like reconstruction pattern of graphene on a copper surface. The image $(12 \mathrm{~nm} \times 12 \mathrm{~nm})$ is taken with a sample bias of $-0.5 \mathrm{~V}$ and tunneling current of $15 \mathrm{pA}$. (b) FFT of the maze-like reconstruction pattern. The six-fold diffraction spots are connected with white lines. The angle between two lattice sets is about $45^{\circ}$.

When the STM tip is located in the bilayer graphene region, Moiré patterns of bilayer graphene with different twist angles have been observed. ${ }^{[27]}$ In the flat region covered with single-layer graphene, a maze-like reconstruction pattern has been observed, as shown in Fig. 2(a). The fast Fourier transform (FFT) of the STM image indicates that the maze-like reconstruction pattern is composed of a six-fold lattice and a four-fold lattice. The lattice constant of the sixfold lattice is $2.5 \pm 0.05 \AA$, which is equal to that of graphene. The four-fold orthogonal lattice constant is $3.6 \pm 0.05 \AA$. The angle between two lattice sets is about $45^{\circ}$, as indicated in Fig. 2(b). We notice that the four-fold symmetry is not perfect since the diffraction spots in one direction are stronger than those in the other direction.

By considering the presence of the graphene and copper substrate in the sample, it is clear that we can assign the six-fold lattice to the single-layer graphene and the four-fold lattice to the copper surface. To understand the interaction between the graphene layer and the underlying copper surface, atomic resolved images of both components are strongly desired, but this is usually very challenging. Fortunately, both the six-fold pattern and the four-fold pattern can be separately imaged with functionalized STM tips. Figures 3(a) and 3(c) show the STM images of the same area with a much clearer six-fold pattern and with a pure orthogonal four-fold pattern, respectively, as well as the FFT of the images. Although the four-fold lattice is still seen in the FFT image of Fig. 3(a), its intensity is significantly reduced as compared with that in Fig. 2(b), and the four-fold symmetry is barely seen in the STM image (Fig. 3(a)). The FFT image for the four-fold pattern (Fig. 3(d)), on the other hand, only shows a set of four-fold diffraction spots. Selectively imaging different electronic states has also been demonstrated in previous STM studies by manipulating the chemical identity at the apex of an STM tip. ${ }^{[28-30]}$ The functionalized STM tips used in our experiment are generated by a gentle poke into the surface or by accidental pick-up of atoms during the scan.
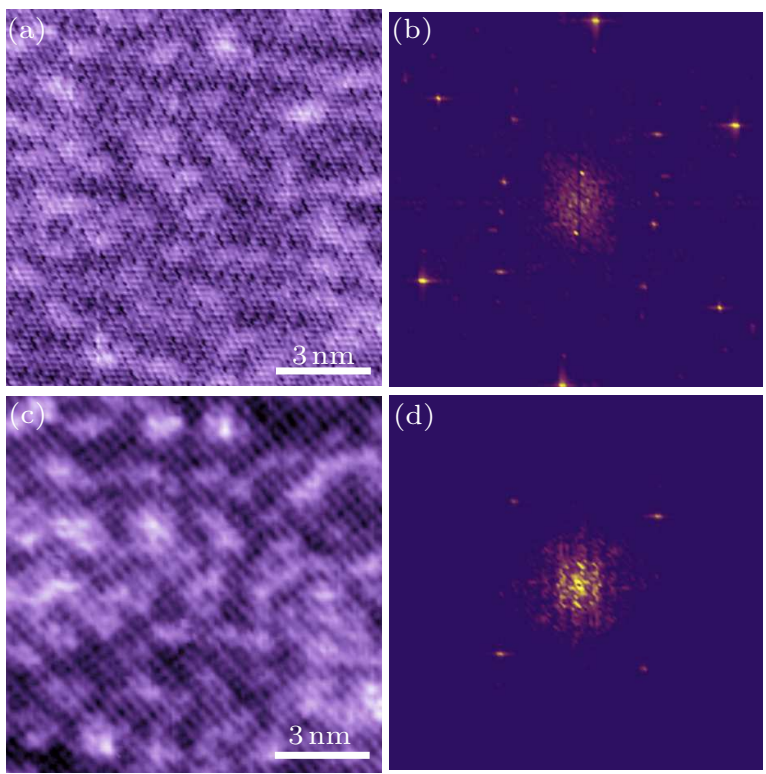

Fig. 3. The STM topographic image (a) and its FFT (b) for the six-fold reconstruction pattern, and the STM topographic image (c) and its FFT (d) for the four-fold reconstruction pattern. The STM images $(12 \mathrm{~nm} \times 12 \mathrm{~nm})$ are taken with functionalized tips in the same area, with a sample bias of $-0.36 \mathrm{~V}$ and tunneling current of $15 \mathrm{pA}$ for (a), and $-0.46 \mathrm{~V}$ and $15 \mathrm{pA}$ for $(\mathrm{c})$.

High-resolution STM images further illustrate the details of both patterns at the atomic scale. As shown in Fig. 4(a), a triangular three-for-six pattern is resolved for the single-layer graphene. In such a structure, three carbon atoms in a hexagonal ring are lifted upward relative to the other three atoms, introducing some $s p^{3}$ hybridization to the otherwise two-dimensional $s p^{2}$ configurations. Previously, such

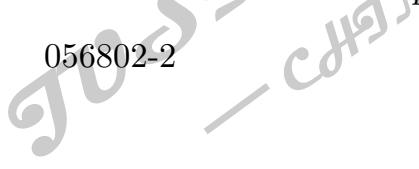


configuration change was observed only in a highly corrugated graphene surface or graphene on $\mathrm{SiO}_{2}$ substrates, ${ }^{[17-19,23]}$ and was attributed to the presence of strong spatially dependent perturbations such as local curvature and trapped charges. The sample surface in our study, however, is flat over $50 \mathrm{~nm}$ and no wrinkles or ripples are observed over a $1 \mu \mathrm{m}$ square area. Thus, the origin of the triangular three-for-six structure observed in our study is distinct from local curvature or trapped charges as reported in previous studies.
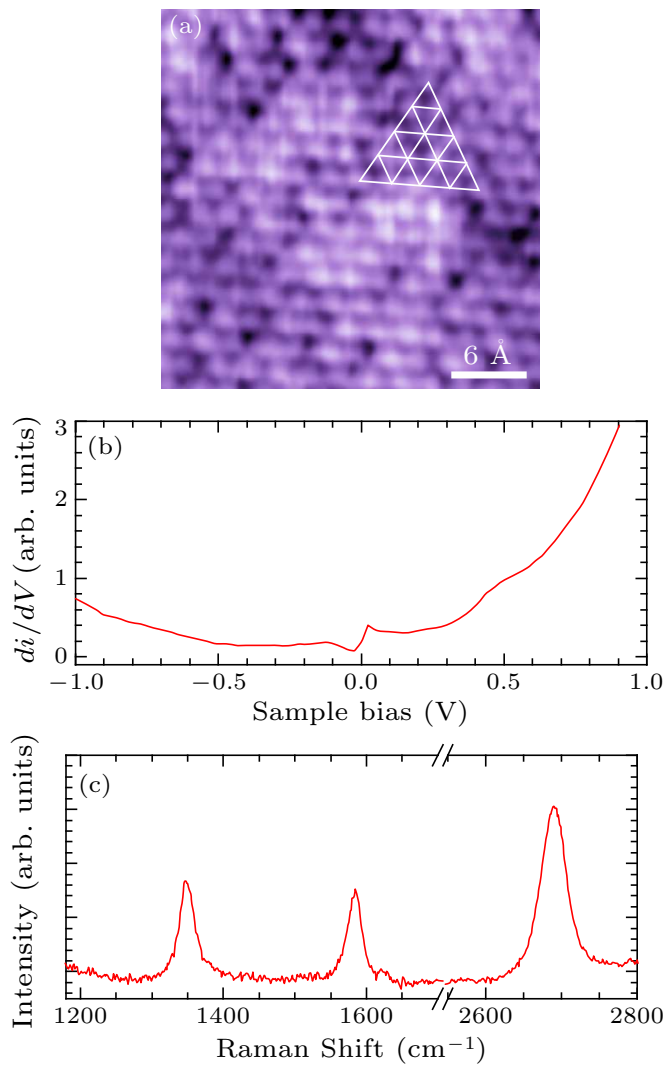

Fig. 4. (a) High-resolution STM image $(3 \mathrm{~nm} \times 3 \mathrm{~nm})$ of the triangular three-for-six pattern of the graphene layer, (b) the $d I / d V$ spectrum of the sample recorded in the single-layer graphene area, and (c) micro-Raman spectroscopy of the sample. The laser wavelength is $532 \mathrm{~nm}$, and the background luminance from the copper substrate has been subtracted.

The emergence of $s p^{3}$ hybridization and the threefor-six reconstruction are further illustrated by the energy-resolved scanning tunneling spectroscopy and micro-Raman spectroscopy. Figure 4(b) presents the $d I / d V$ spectrum of the sample by scanning sample bias from $-1 \mathrm{~V}$ to $1 \mathrm{~V}$ relative to the tip. The Dirac point is located at about $0.4 \mathrm{eV}$ below the Fermi energy, indicating the charge transfer from the substrate to the graphene. A depression of LDOS is observed at zero bias due to the lack of excitation of the optical phonon mode at a very low energy in the graphene. ${ }^{[12,15]}$ By analyzing the second derivative spectrum, we find that the phonon energy is about $69 \mathrm{meV}$, which is close to the observation in Ref. [12].
In addition, a sharply increasing LDOS that is almost above zero bias is observed in the $d I / d V$ curve as well. The exact nature of this state is not yet clear. Recent theoretical calculations predict that quasi-bound states localized around defects can induce a bump in LDOS above the Dirac point in graphene. ${ }^{[32]}$ Wehling et al. stated that a midgap state appears as a universal feature of monovalent impurities bonded to graphene's carbon atoms. ${ }^{[31]}$ The midgap states may also be formed due to vacancies, cracks, boundaries, or impurities in the substrate with a high potential difference with respect to the graphene sheet. On the other hand, the homogenous three-to-six pattern in Fig. 4(a) suggests that $s p^{3}$ hybridization is not such a local effect as that induced by nitrogen doping. ${ }^{[14]}$ Thus, one possible cause of such a state is the bonding between copper atoms in the reconstructed surface and carbon atoms in graphene. To determine the details of such a state, further experiments and theoretical calculation have to be carried out. Nonetheless, our tunneling spectroscopy suggests a significant interaction between the graphene single layer and the reconstructed copper surface, where the copper atoms underneath the graphene play a similar role to the defects in introducing $s p^{3}$ hybridization and generating an electronic state near the Fermi level.
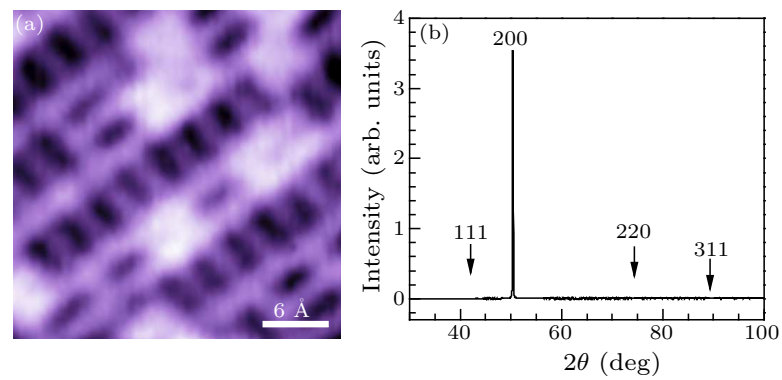

Fig. 5. (a) High-resolution STM image $(3 \mathrm{~nm} \times 3 \mathrm{~nm})$ of the four-fold pattern, and (b) the X-ray diffraction pattern of the sample surface.

The existence of strong perturbation from the substrate is also suggested by the micro-Raman spectrum as shown in Fig.4(c). A strong resonance peak of the $\mathrm{D}$ band at $1347 \mathrm{~cm}^{-1}$ and a weak $\mathrm{D}^{\prime}$ band at $1620 \mathrm{~cm}^{-1}$ are observed, and these are usually associated with the presence of lattice distortion, defects, or edge states in single-layer graphene. ${ }^{[25,33]}$ The measured high intensity of the D band, comparable to the intensity of the $\mathrm{G}$ band, is believed to be due to the three-for-six structure conformation of the graphene in our sample. The $2 \mathrm{D}$ band presents a very sharp single resonance peak with an intensity greater than the $G$ resonance, which indicates the existence of a high-quality single-layer graphene.

Figure 5(a) shows a high-resolution STM image of the four-fold orthogonal pattern, which identifies the reconstruction with the aid of an x-ray diffraction pattern of the sample surface. The x-ray diffraction pattern, measured after the STM experiment, 
shows a dominant crystal surface orientation along the [100] direction (Fig. 5(b)). It has been reported that copper foil crystallizes during the growth process in the CVD oven, ${ }^{[34]}$ since a growth temperature of $1050^{\circ} \mathrm{C}$ is high enough to induce the crystallization, and graphene can play a significant role during the crystallization and surface reconstruction. Taking the fact that the distance between adjacent atoms on the $\mathrm{Cu}(100)$ surface is $2.55 \AA$, the observed orthogonal pattern with a lattice constant of $3.6 \pm 0.05 \AA$ is then determined as a mixed $(2 \sqrt{2} \times \sqrt{2}) R 45^{\circ}$ reconstruction on the $\mathrm{Cu}(100)$ surface. A similar mixed reconstruction was observed with the chemisorption of oxygen on the $\mathrm{Cu}(100)$ crystal surface, as revealed by low-energy electron diffraction (LEED) and STM studies. ${ }^{[35,36]}$ For the graphene-covered copper surface as in our study, the carbon atom in graphene is suggested to play a similar role as the chemisorbed oxygen in surface reconstruction, which results in a strong interaction between graphene and the copper substrate.
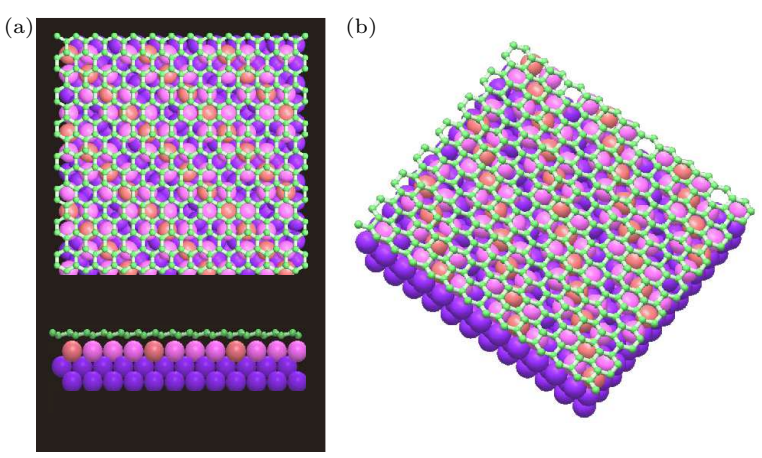

Fig. 6. Atomic model of the reconstructed graphenecopper system. The graphene is shown with green balls and sticks. The copper atom of the $(2 \sqrt{2} \times \sqrt{2}) R 45^{\circ}$ reconstruction is represented by red and pink balls, while the copper atoms under the top layer are blue.

An atomic schematic model was constructed based on the atomic-resolved information, as shown in Fig. 6. The upper part on the left is a view from the top, while the lower part is a view from the side and a 3D model is presented on the right. In the graphene overlayer, three atoms in a hexagonal ring are pulled towards the substrate to form the three-for-six configuration. The structure of the copper substrate is constructed following the missing-row model by Zeng et al., ${ }^{[35]}$ except that oxygen atoms are removed for clarity. The copper atom of the $(2 \sqrt{2} \times \sqrt{2}) R 45^{\circ}$ reconstruction is represented by red and pink balls. We are not able to exclude the existence of oxygen atoms in such a sample system, even though the oxide layer can be removed during annealing. ${ }^{[34]}$

We note that UHV annealing may play a significant role in enhancing the graphene-copper interaction and generating the observed novel structure pattern in our sample system. The Raman spectroscopy on the as-grown sample without UHV annealing shows no peak of $\mathrm{D}$ band resonance, indicating a weak perturbation from the substrate. Recent STM studies on similar graphene systems reported stripe and rhombic patterns of graphene, ${ }^{[34,37]}$ where no apparent three-for-six pattern was observed and a weak graphene-substrate interaction was concluded. The nature of various experimentally observed structures in the graphene-substrate system and the role of UHV annealing in modulating graphene-substrate interaction needs further investigation, with both experimental and theoretical calculations.

In summary, prominent interaction between singlelayer graphene and a copper substrate has been demonstrated in a maze-like reconstruction pattern. The triangular three-for-six structure in graphene and a mixed $(2 \sqrt{2} \times \sqrt{2}) R 45^{\circ}$ reconstruction of the $\mathrm{Cu}(100)$ surface are revealed with functionalized STM tips. The electronic property of the graphene is modified as a result of partial $s p^{3}$ hybridization. Besides the methods of introducing external impurity and internal structure defects, our results suggest another effective way to induce $s p^{3}$ hybridization and thus to tune the electronic properties of a single-layer graphene through its interaction with the substrates.

XHL would like to thank Professor Sheng Meng (Institute of Physics, Chinese Academy of Sciences) for helpful discussions.

\section{References}

[1] Novoselov K S et al 2004 Science 306666

[2] Novoselov K S et al 2005 Nature 438197

[3] Zhang Y et al 2005 Nature 438201

[4] Berger C et al 2006 Science 3121191

[5] Geim A K and Novoselov K S 2007 Nat. Mater. 6183

[6] Kim K S et al 2009 Nature 457706

[7] Li X et al 2009 Science $\mathbf{3 2 4} 1312$

[8] Li X S et al 2009 Nano Lett. 94359

[9] Novoselov K S et al 2012 Nature 490192

[10] Wallace P R 1947 Phys. Rev. 71622

[11] Castro Neto A H et al 2009 Rev. Mod. Phys. 81109

[12] Zhang Y et al 2008 Nat. Phys. 4627

[13] Balog R et al 2010 Nat. Mater. 9315

[14] Zhao L et al 2011 Science 333999

[15] Elias D C et al 2009 Science $\mathbf{3 2 3} 610$

[16] Ryu S et al 2008 Nano Lett. 84597

[17] Ishigami M et al 2007 Nano Lett. 71643

[18] Xu K, Cao P and Heath J R 2009 Nano Lett. 94446

[19] Zhang Y et al 2011 ACS Nano 54014

[20] Zhou S Y et al 2007 Nat. Mater. 6770

[21] Rotenberg E et al 2008 Nat. Mater. 7258

[22] Zhou S Y et al 2008 Nat. Mater. 7259

[23] Stolyarova E et al 2007 Proc. Natl. Acad. Sci. USA 104 9209

[24] Wu W et al 2010 Sens. Actuators B 101016

[25] Wu W et al 2011 Adv. Mater. 234898

[26] Yu Q et al 2011 Nat. Mater. 10443

[27] Wang Y et al 2013 Nat. Commun. (submitted)

[28] Deng Z T et al 2006 Phys. Rev. Lett. 96156102

[29] Hamers R J, Tromp R M and Demuth J E 1986 Phys. Rev. Lett. 561972

[30] Ruan L et al 1993 Phys. Rev. Lett. 704079

[31] Wehling T O, Katsnelson M I and Lichtenstein A I 2009 Phys. Rev. B $\mathbf{8 0} 085428$

[32] Lherbier A S et al 2011 Phys. Rev. Lett. 106046803

[33] Ferrari A C et al 2006 Phys.Rev. Lett. 97187401

[34] Cho J et al 2011 ACS Nano 53607

[35] Zeng H C, McFarlane R A and Mitchell K A R 1989 Surf. Sci. 208 L7

[36] Woll Ch et al 1990 Phys. Rev. B 4211926

[37] Tian J et al 2012 Nano Lett. 123893 


\title{
Chinese Physics Letters
}

\author{
Volume $30 \quad$ Number $5 \quad$ May 2013
}

\section{GENERAL}

050301 Charge States and Transition of Double Quantum Dot in the Few-Electron Regime ZHOU Cheng, WANG Li, TU Tao, HAN Tian-Yi, LI Hai-Ou, GUO Guo-Ping

050302 The Strategic Form of Quantum Prisoners' Dilemma Ahmad Nawaz

050303 Sudden Transition in Quantum Discord Dynamics: Role of Three-Site Interaction TIAN Li-Jun, ZHANG Cai-Yun, QIN Li-Guo

050304 Preparation of $N$-Qubit GHZ State with a Hybrid Quantum System Based on Nitrogen-Vacancy Centers ZHAO Yu-Jing, FANG Xi-Ming, ZHOU Fang, SONG Ke-Hui

050501 Local Property of Recurrence Network for Investigating Gas-Liquid Two-Phase Flow Characteristics ZHANG Xin-Wang, JIN Ning-De, GAO Zhong-Ke, ZHAI Lu-Sheng

050701 Relations between Mass Change and Frequency Shift of a QCM Sensor in Contact with Viscoelastic Medium TAN Feng, HUANG Xian-He

\section{THE PHYSICS OF ELEMENTARY PARTICLES AND FIELDS}

051201 The Quark Number Susceptibility of QCD at Finite Temperature and Chemical Potential ZHU Hui-Xia, SUN Wei-Min, ZONG Hong-Shi

051301 Jet Energy Shift due to Non-Perturbative QCD Effects in p+p Collisions Studied with PYTHIA

LI Han-Lin, ZHANG Ben-Wei, WANG En-Ke

\section{NUCLEAR PHYSICS}

052101 Spin-Flip Response Function of Finite Nuclei in a Fully Self-Consistent RPA Approach WEN Pei-Wei, CAO Li-Gang

052102 Bound $0^{+}$Excited States of Three-Body Systems with Short-Range Two-Body Interactions LIN Qi-Hu, REN Zhong-Zhou

052103 The Nuclear Incompressibility and Isoscalar Giant Dipole Resonance in Relativistic Continuum Random Phase Approximation YANG Ding, CAO Li-Gang, MA Zhong-Yu

052501 Re-examination of Finite-Size Effects in Isobaric Yield Ratios Using a Statistical Abrasion-Ablation Model MA Chun-Wang, WANG Shan-Shan, WEI Hui-Ling, MA Yu-Gang

052901 Tuning and Cold Test of a Four-Vane RFQ with Ramped Inter-Vane Voltage for the Compact Pulsed Hadron Source XING Qing-Zi, DU Lei, ZHENG Shu-Xin, GUAN Xia-Ling, LI Jian, CAI Jin-Chi, GONG Cun-Kui WANG Xue-Wu, TANG Chuan-Xiang, James Billen, James Stovall, Lloyd Young

\section{ATOMIC AND MOLECULAR PHYSICS}

053601 The Electronic Structures and Optical Properties of Substituted Rare-Earth Manganite $\mathrm{Tb}_{1-x} \mathrm{Yb}_{x} \mathrm{MnO}_{3}$ CAI Lu-Gang, LIU Fa-Min, ZHONG Wen-Wu, ZHANG Dian 


\section{FUNDAMENTAL AREAS OF PHENOMENOLOGY(INCLUDING}

APPLICATIONS)

054202 Single-Photon Transmission Characteristics in a Pair of Coupled-Resonator Waveguides Linked by a Nanocavity Containing a Quantum Emitter CHENG Mu-Tian, DING Meng-Ting, SONG Yan-Yan, LUO Ya-Qin

054203 Generalized Ptychography with Diverse Probes SHI Yi-Shi, WANG Ya-Li, ZHANG San-Guo

054204 Measurement of Long-Pulse Fine Structure by Time-Spectrum Coding LIU Ji-Gang, XIE Guo-Qiang, QIAN Lie-Jia, YUAN Peng

054205 A Multiple Phase-Shifted Distributed Feedback (DFB) Laser Fabricated by Nanoimprint Lithography ZUO Qiang, ZHAO Jian-Yi, CHEN Xin, WANG Zhi-Hao, SUN Tang-You, ZHOU Ning, ZHAO Yan-Li, XU Zhi-Mou, LIU Wen

054206 Improvement of the Focusing Resolution of Photonic Crystal Negative Refraction Imaging with a Hollow Component Structure CHEN Shou-Xiang, YANG Xiu-Lun, MENG Xiang-Feng, DONG Guo-Yan, WANG Yu-Rong, WANG Lin-Hui, HUANG Zhe

054207 Efficient $1.8 \mu \mathrm{m}$ Emission of a Novel $\mathrm{Tm}^{3+}$ Doped Germanate-Tellurite Glass PENG Ya-Pei, GUO Yan-Yan, ZHANG Jun-Jie

054208 An All Fiber $1.94 \mu \mathrm{m}$ Nanosecond Pulse Laser Amplified by Highly Tm$^{3+}{ }_{\text {-Doped Silicon }}$ Fibers ZHOU Ren-Lai, REN Jian-Cun, YANG Chao, JU You-Lun, WANG Yue-Zhu

054209 Super-Resolved Digital Holography Based on the Coherent Diffraction Imaging Scheme WANG Bao-Sheng, PAN Xing-Chen, WANG Hai-Yan, CHENG Jun, GAO Shu-Mei, LIU Cheng, ZHU Jian-Qiang

054210 A Passively Mode-Locked Erbium-Doped Fiber Laser Based on a Single-Wall Carbon Nanotube Polymer F. Ahmad, S. W. Harun, R. M. Nor, N. R. Zulkepely, H. Ahmad, P. Shum

054211 Spatial Correlation Properties of Tightly Focused $J_{0}$-Correlated Azimuthally Polarized Vortex Beams RAO Lian-Zhou, LIN Hui-Chuan, SUN Qing-Quan

054212 High-Resolution Compact Plasmonic Wavelength Demultiplexers Based on Cascading Square Resonators CHEN Zhao, YU Li, WANG Lu-Lu, ZHAO Yu-Fang, DUAN Gao-Yan, XIAO Jing-Hua

054301 Suppression of Natural Convection in a Thermoacoustic Pulse Tube Refrigerator HAN Jun-Qing, LIU Qiu-Sheng

054302 Sound Velocity in Water and Ice up to $4.2 \mathrm{GPa}$ and $500 \mathrm{~K}$ on Multi-Anvil Apparatus WANG Zhi-Gang, LIU Yong-Gang, ZHOU Wen-Ge, SONG Wei, BI Yan, LIU Lei, XIE Hong-Sen

054701 Deflagration-to-Detonation Transition Induced by Hot Jets in a Supersonic Premixed Airstream HAN Xu, ZHOU Jin, LIN Zhi-Yong, LIU Yu

PHYSICS OF GASES, PLASMAS, AND ELECTRIC DISCHARGES

055201 Physical Process for the Pick-Up of Minor Ions by Low-Frequency Alfvén Waves WANG Chuan-Bing, WEI Jing-Dong, WANG Bin, WANG Shui

CONDENSED MATTER: STRUCTURE, MECHANICAL AND THERMAL PROPERTIES

056201 Embedded Atom Method-Based Geometry Optimization Aspects of Body-Centered Cubic Metals M. Güler, E. Güler 
056801 The Formation and Characterization of GaN Hexagonal Pyramids

ZHANG Shi-Ying, XIU Xiang-Qian, LIN Zeng-Qin, HUA Xue-Mei, XIE Zi-Li, ZHANG Rong, ZHENG You-Dou

056802 Visualization of a Maze-Like Reconstruction of Graphene on a Copper Surface at the Atomic Scale

XIE Nan, GONG Hui-Qi, ZHOU Zhi, GUO Xiao-Dong, YAN Shi-Chao, SUN Qian, XING Sirui, WU Wei, PEI Shin-shem, BAO Jiming, SHAN Xin-Yan, GUO Yang, LU Xing-Hua

CONDENSED MATTER: ELECTRONIC STRUCTURE, ELECTRICAL, MAGNETIC, AND OPTICAL PROPERTIES

057101 The Valence Band Offset of an $\mathrm{Al}_{0.17} \mathrm{Ga}_{0.83} \mathrm{~N} / \mathrm{GaN}$ Heterojunction Determined by X-Ray Photoelectron Spectroscopy

WAN Xiao-Jia, WANG Xiao-Liang, XIAO Hong-Ling, WANG Cui-Mei, FENG Chun, DENG Qing-Wen, QU Shen-Qi, ZHANG Jing-Wen, HOU Xun, CAI Shu-Jun, FENG Zhi-Hong

057201 A Spin-Polarized Current Direction Controller Based on a Nonuniform Rashba Quantum Wire XU Zhong-Hui, XIAO Wen, CHEN Yu-Guang

057301 Fano Resonance Based on Multimode Interference in Symmetric Plasmonic Structures and its Applications in Plasmonic Nanosensors

CHEN Zong-Qiang, QI Ji-Wei, CHEN Jing, LI Yu-Dong, HAO Zhi-Qiang, LU Wen-Qiang, XU Jing-Jun, SUN Qian

057302 The Performance Characteristics of a Nano-thermoelectric Refrigerator Driven by an External Stochastic Force WANG Hao, WU Guo-Xing

057303 GaN Schottky Barrier Diodes with High-Resistivity Edge Termination Formed by Boron Implantation XU Wei-Zong, FU Li-Hua, LU Hai, REN Fang-Fang, CHEN Dun-Jun, ZHANG Rong, ZHENG You-Dou

057401 The Generalized Joint Density of States and Its Application to Exploring the Pairing Symmetry of High- $T_{\mathrm{c}}$ Superconductors ZHANG Dan-Bo, HAN Qiang, WANG Zi-Dan

057701 Ferroelectricity in Pulsed Laser Deposited $\mathrm{Ba}\left(\mathrm{Fe}_{1 / 2} \mathrm{Nb}_{1 / 2}\right) \mathrm{O}_{3}$ Thin Films ZHANG Wei, WU Shu-Ya, LI Lei, CHEN Xiang-Ming

057801 The Metal Intermediate Electrode: A Method to Improve the Current Efficiency of a Tandem Organic Light-Emitting Diode ZHANG Yan-Fei, ZHAO Su-Ling, XU Zheng

057802 The Analysis of Main Dosimetric Glow Peaks in $\mathrm{CaF}_{2}: \mathrm{Tm}$ (TLD-300)

Vural E. Kafadar, Metin Bedir, A. Necmeddin Yazıcı, Tülin Günal

057803 Optical Properties of CdS/PVA Nanocomposite Films Synthesized using the Gamma-Irradiation-Induced Method

Alireza Kharazmi, Elias Saion, Nastaran Faraji, Nayereh Soltani, Arash Dehzangi

057804 Plastic Deformation of Nanocrystalline Zinc Investigated by Positron Annihilation Lifetime Spectroscopy

ZHOU Kai, LI Hui, WANG Zhu

CROSS-DISCIPLINARY PHYSICS AND RELATED AREAS OF SCIENCE AND TECHNOLOGY

$058101 \mathrm{GeTe}_{4}$ as a Candidate for Phase Change Memory Application

LI Run, TANG Shi-Yu, BAI Gang, YIN Qiao-Nan, LAN Xue-Xin, XIA Yi-Dong, YIN Jiang, LIU Zhi-Guo

058501 Measurement of the Internal Loss Coefficient of Semiconductor Optical Amplifiers

LIU Guo-Dong, WU Chong-Qing, WANG Fu, MAO Ya-Ya

058502 InAlN/AlN/GaN Field-Plated MIS-HEMTs with a Plasma-Enhanced Chemical Vapor Deposition SiN Gate Dielectric

MAO Wei, HAO Yue, YANG Cui, ZHANG Jin-Cheng, MA Xiao-Hua, WANG Chong, LIU Hong-Xia, YANG Lin-An, ZHANG Jin-Feng, ZHENG Xue-Feng, ZHANG Kai, CHEN Yong-He, YANG Li-Yuan 
058503 InGaN-Based Blue Light Emitting Diodes with AlInN-GaN-AlInN Electron Blocking Layers TONG Jin-Hui, ZHAO Bi-Jun, REN Zhi-Wei, WANG Xing-Fu, CHEN Xin, LI Shu-Ti

058901 Statistics of Leaders in Index-Driven Networks

ZHANG Yong, JU Xian-Meng, ZHANG Li-Jie, XU Xin-Jian

\section{GEOPHYSICS, ASTRONOMY, AND ASTROPHYSICS}

059401 Counter-Streaming Interaction between Fast Magnetosonic Wave and Radiation Belt Electrons

ZHU Hui, SU Zhen-Peng, ZHENG Hui-Nan

059501 Differentiating Neutron Star Models by X-Ray Polarimetry

LU Ji-Guang, XU Ren-Xin, FENG Hua

059701 The Dynamical Effects of a Large-Scale Ordered Magnetic Field on Slim Disks PING Chong, MA Ren-Yi, LU Ju-Fu 